Self-Assembly

\title{
Spatially Oriented and Reversible Surface Assembly of Single-Walled Carbon Nanotubes: A Strategy Based on $\boldsymbol{\pi}-\boldsymbol{\pi}$ Interactions**
}

\author{
Tommaso Ferri, * Daniele Frasca, Olimpia Arias de Fuentes, Roberto Santucci, and \\ Marco Frasconi*
}

The unique structural, mechanical, and electronic properties of carbon nanotubes (CNTs) have resulted in their development into a standard material for a variety of applications. In particular, CNTs have been widely investigated for electronic applications, which include use as transistors ${ }^{[1]}$ photovoltaic devices, ${ }^{[2]}$ active sensing elements, ${ }^{[3]}$ and building blocks for biosensors. ${ }^{[4]}$ One of the main challenges currently involves assembling CNTs on a substrate in a manner that allows the secondary association of redox-active components to the CNTs and rapid electron transfer along the tubes. ${ }^{[4]}$ To this end, functional groups are formed at the CNT edges in order to ensure stable anchoring. ${ }^{[5,6]}$ Alternative routes exploit the physical adsorption of either hydrophilic groups ${ }^{[7]}$ or watersoluble polymers on the CNT walls. ${ }^{[8]}$ Although polymercoated CNTs were used for the functionalization of different surfaces ${ }^{[8]}$ this method has severe limitations when fabricating highly oriented single-walled CNT (SWCNT) arrays. Indeed, the ability to prepare vertically aligned SWCNTs is a crucial point for improving the performance of nanodevices. ${ }^{[9]}$

Covalent attachment of vertically aligned SWCNTs is usually achieved by amide linkages. ${ }^{[10]}$ Recently, we reported the formation of dense arrays of SWCNT "forests" on different substrates by using diazonium salt coupling. ${ }^{[11]}$ Nevertheless, a valid alternative is represented by the noncovalent direct immobilization of SWCNTs on a surface, since in this case no structural alteration of the SWCNTs

[*] Prof. T. Ferri, Dr. D. Frasca

Dipartimento di Chimica

Università di Roma "La Sapienza"

Piazzale Aldo Moro 5, Roma 00185 (Italy)

E-mail: tommaso.ferri@uniroma1.it

Dr. M. Frasconi ${ }^{[+]}$

Dipartimento di Chimica e Tecnologie del Farmaco

Università di Roma "La Sapienza"

Piazzale Aldo Moro 5, Roma 00185 (Italy)

E-mail:marco.frasconi@uniroma1.it

Prof. O. Arias de Fuentes

Instituto de Ciencia y Tecnología de Materiales

Universidad de La Habana, Ciudad de La Habana (Cuba)

Prof. R. Santucci

Dipartimento di Medicina Sperimentale e Scienze Biochimiche Università di Roma "Tor Vergata", Roma (Italy)

$\left[^{+}\right]$Present address: Department of Chemistry Northwestern University

2145 Sheridan Road, Evanston, IL 60208-3113 (USA)

[***] We gratefully acknowledge $H$. Nizri for support with the AFM measurements and A. D'Annibale for providing biphenylthiol.

밍 Supporting information for this article is available on the WWW under http://dx.doi.org/10.1002/anie.201102406. occurred ${ }^{[12]}$ In view of the growing interest in the construction of simple and cost-effective systems, we report herein a new strategy to achieve a spatially controlled SWCNT assembly on a gold surface by exploiting $\pi-\pi$ interactions. To the best of our knowledge, no report on the oriented and reversible assembly of SWCNTs on a surface by using noncovalent $\pi-\pi$ stacking interactions has been reported to date. In particular, the proposed method is based on the spontaneous adsorption of SWCNTs on self-assembled monolayers (SAMs) of suitable aromatic molecules. To this end, a stable dispersion of purified SWCNTs (HiPCO) in DMF was obtained by sonication, as previously described. ${ }^{[13]}$ The average length of the suspended SWCNTs was $60-120 \mathrm{~nm}$, as evaluated by transmission electron microscopy (TEM).

The adsorption of SWCNTs was achieved by immersing $\mathrm{Au}$ surfaces that were modified with SAMs of phenylthiol (PT), 4,4'-dithiobipyridine (DTBPY), and biphenylthiol (BPT) overnight in a suspension of SWCNTs. The adsorption kinetics were investigated by using a quartz crystal microbalance (QCM). The frequency changes $(\Delta f)$ of the Au/quartz crystal modified with different SAMs increased upon the immobilization of SWCNTs (Figure S1 in the Supporting Information). After the adsorption was complete (ca. $90 \mathrm{~min}$ ), we estimated that approximately $3.4 \pm 0.2$ $(\Delta f=-572 \mathrm{~Hz}), \quad 3.9 \pm 0.1 \quad(\Delta f=-658 \mathrm{~Hz}), \quad$ and $\quad 6.7 \pm 0.2$ $(\Delta f=-1125 \mathrm{~Hz}) \mu \mathrm{g} \mathrm{cm}^{-2}$ of SWCNTs were present on the surface modified with PT, DTBPY, and DPT, respectively. From the average length, the mass of adsorbed SWCNTs, and the electrode area, the determined SWCNT densities on PT and DTBPY-modified surfaces were approximately $3 \times 10^{13}$ and $5 \times 10^{13} \mathrm{NTs} \mathrm{cm}^{-2}$, respectively.

The SWCNT assembly on SAM-modified surfaces was followed by atomic force microscopy (AFM). Isolated protrusions were observed on the surface after $20 \mathrm{~min}$ of surface modification (Figure S2), the density of which increases gradually, until densely packed monolayers of standing SWCNTs assembled on different SAMs were observed (Figure $1 \mathrm{a}-\mathrm{c}$ ). The surface roughness increases up to $120 \mathrm{~min}$ and then decreases as the layer of SWCNTs "fills in". Needle-like patterns of vertically aligned SWCNTs with a maximum height of $50-60 \mathrm{~nm}$ were obtained on PT and DTBPY SAMs, whereas on BPT SAMs, the anchored SWCNTs were found to be slightly higher (ca. $80 \mathrm{~nm}$ ), as evaluated by imaging a step region between the assembled SWCNTs and the bare gold surface (Figure S3). We hypothesize that the assembly process "favors" shorter SWCNTs because they diffuse more quickly onto the surface.

A successive inspection of the AFM images revealed the presence of SWCNT bundles normal to the BPT-modified 

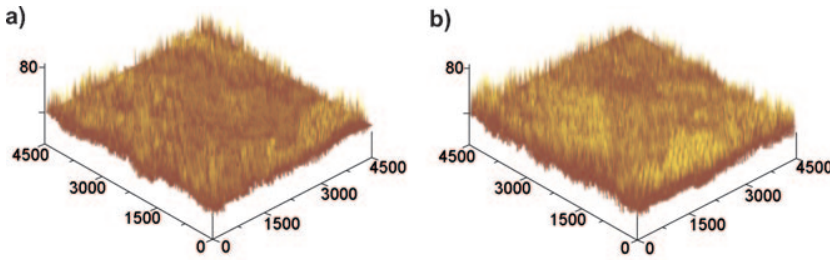

c)
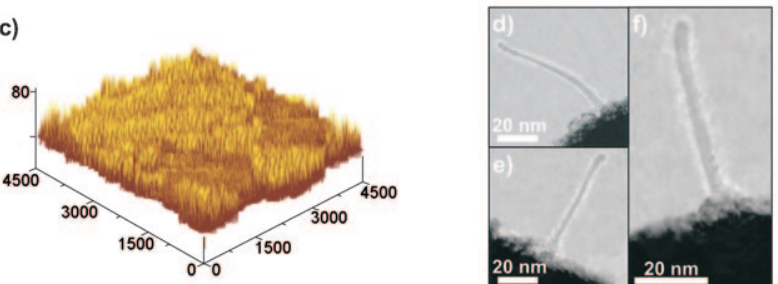

Figure 1. AFM images of SWCNTs assembled on a) PT, b) DTBPY, and c) BPT monolayers associated with a gold surface after $120 \mathrm{~min}$ adsorption. TEM images of SWCNTs adsorbed on an ultrathin gold wire modified with d) PT, e) DTBPY, and f) BPT monolayers. All dimensions are in $\mathrm{nm}$.

surface. This observation, together with the weight coverage obtained by QCM experiments, suggests that a densely packed monolayer of SWCNTs is associated with the SWCNT assembly on a BPT monolayer. This behavior may arise as the $\pi-\pi$ interactions between the hydrophobic sidewalls of the SWCNTs and the biphenyl functionalities on the surface are stronger than those with the monolayers of PT and DTBPY. High-resolution TEM was employed to determine the orientation of SWCNTs assembled on a SAMmodified ultrathin wire. The results clearly show a vertical alignment of the SWCNTs tethered by only one end to the gold surface (Figure 1d-f).

The evidence of the role of $\pi-\pi$ interactions between the aromatic rings anchored on the surface and the SWCNTs in determining a vertical alignment of SWCNTs may be gathered by comparing the assembly of the SWCNTs on the proposed aromatic SAMs with the adsorption on an alkanethiol. The topography of AFM image (Figure S4) clearly shows a completely different alignment of SWCNTs adsorbed on an octadecanthiol SAM. The $\pi-\pi$ interactions between the hydrophobic sidewalls of the SWCNTs and the aromatic rings on the gold surface determine the orientation of the SWCNTs on the surface.

Further support of the vertical alignment of SWCNTs was obtained by polarized Raman spectroscopy. The signal intensity in all investigated Raman modes strongly depends on the SWCNT orientation ${ }^{[14]}$ the maximum signal is obtained when the polarization of the incident light is parallel to the CNT axis (Figure 2a) and it is greatly reduced when light is perpendicularly polarized (Figure $2 \mathrm{~b}$ ). This result implies that the SWCNTs are oriented normal to the BPTmodified gold substrate. A similar orientation dependence of the Raman modes was observed for SWCNTs assembled on PT and DTBPY SAMs (Figure S5).

To gain further information on the adsorption kinetics, the SWCNT immobilization was monitored at different temperatures by surface plasmon resonance (SPR) spectroscopy. The shift of the SPR signal is associated with the adsorption of

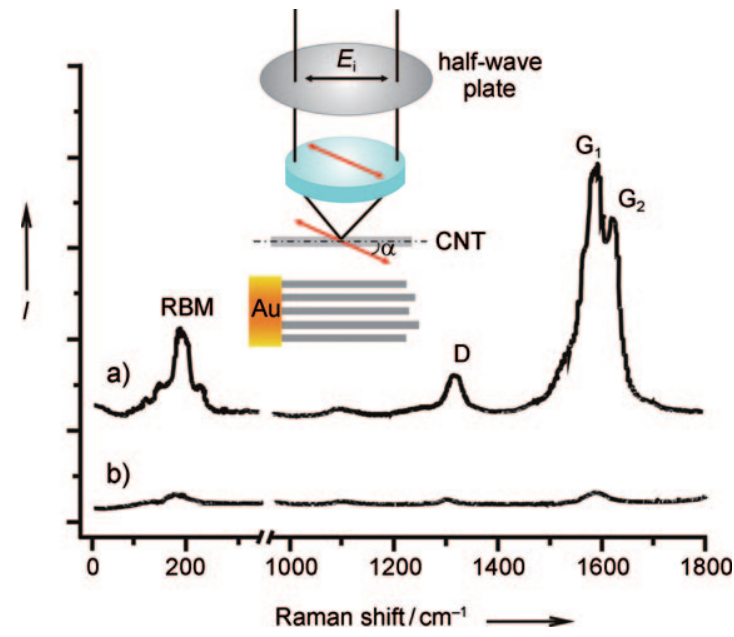

Figure 2. Raman spectra at the cross-section of the BPT-gold substrate coated with SWCNTs for a) parallel and b) perpendicular angles $(\alpha)$ between the nanotube axis and the polarization of the incident laser beam, as shown in the inset. RBM= radial breathing mode.

SWCNTs on SAMs. The process is governed by Langmuirtype adsorption kinetics, as shown by the good correlation between the experimental and fitting curves (Figure S6). A faster adsorption rate was observed at higher temperatures, and is consistent with the stronger Brownian motion of SWCNTs that occurs in solution, resulting in a higher probability of the SWCNTs-SAMs interaction. ${ }^{[15]}$ Both the adsorption $\left(k_{\mathrm{a}}\right)$ and desorption $\left(k_{\mathrm{d}}\right)$ rate constants for the SAMs were determined from the fitting curves; values of $0.0017,0.0012$, and $0.0039\left(k_{\mathrm{a}} \mathrm{C}+k_{\mathrm{d}}\right)$ were determined for the SWCNT adsorption on surfaces modified with PT, DTBPY, and BPT, respectively. In the latter case, the consistent stability of the system can be ascribed to the stronger $\pi-\pi$ stacking interactions between the hydrophobic side walls of the SWCNTs and the biphenyl rings assembled on the surface.

The electron transfer (eT) properties of the SWCNT assembly are crucial in view of possible applications in the field of electronics. Therefore, the electrochemical behavior of a bare and SAM-modified Au surface was compared with that of a SWCNT-covered surface. To this end, cyclic voltammograms $(\mathrm{CVs})$ of the $\mathrm{Fe}(\mathrm{CN})_{6}{ }^{3-/ 4-}$ couple in $1 \mathrm{M} \mathrm{KCl}$ were obtained (Figure S7). Quasi-reversible CVs were recorded at a bare $\mathrm{Au}$ electrode, and an eT rate constant $\left(k_{\mathrm{ET}}\right)$ of $0.10 \mathrm{~cm} \mathrm{~s}^{-1}$ was calculated from the peak separation. ${ }^{[16]}$ A decrease of the process reversibility was observed after the SAMs were assembled on the electrode surface $\left(k_{\mathrm{ET}}=0.03 \mathrm{~cm} \mathrm{~s}^{-1}\right)$. This observation suggests that the SAMs act as a barrier that opposes a close approach of the redox marker to the electrode. The adsorption of SWCNTs on a SAM-modified surface significantly increases the heterogeneous eT rate: $k_{\mathrm{ET}}$ values of $0.14,0.13$, and $0.09 \mathrm{~cm} \mathrm{~s}^{-1}$ were determined for SWCNTs on PT, DTBPY, and BPT SAMs, respectively. Similar to previously observed behavior, ${ }^{[9,10 \mathrm{~g}]}$ the tether layers indeed control the eT between the SWCNTs and the electrode, and this affects the overall eT process. Adsorption of SWCNTs on a fully conjugated layer strongly increases the eT rate across the interface, and thus has important implications for possible electronic applications. 
In view of the interesting potential of CNT-array-based devices for applications in the field of biotechnology, the reusability of the system is critical for the development of cost-effective devices. To produce a substrate that is suitable for repeated use, SWCNTs should be removed from the surface without affecting the SAM, and the system based on DTBPY appears to be the most suitable. The $\mathrm{p} K_{\mathrm{a}}$ value of the pyridine unit is 5.2; thus, pyridine is in the neutral state at $\mathrm{pH}>5.2$, whereas it exists in the protonated form at $\mathrm{pH}<5.2$. In the neutral form, the monolayer is expected to favor SWCNT assembly because of stabilization of the $\pi-\pi$ stacking interactions; conversely, in the protonated form, the monolayer destabilizes the pyridine-SWCNTs $\pi-\pi$ interactions, thus facilitating the release of the SWCNTs from the surface. The AFM images in Figure 3 show that upon treatment with

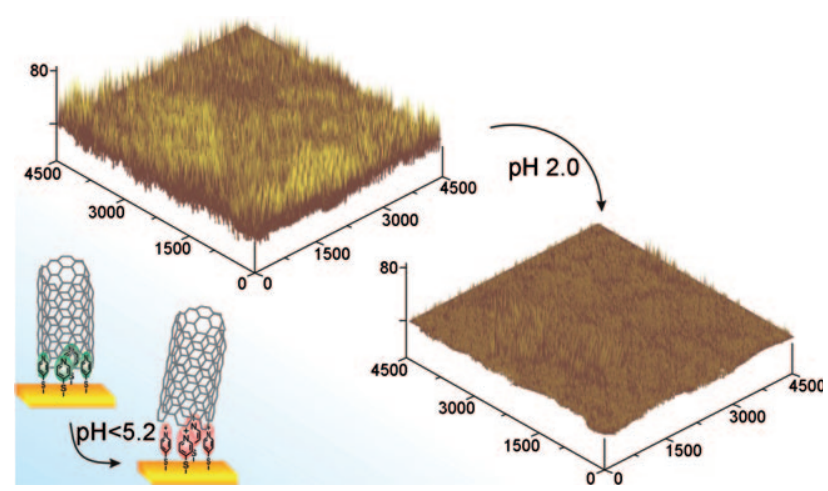

Figure 3. $\mathrm{pH}$-controlled desorption of SWCNTs from a DTBPY-functionalized gold surface, and corresponding AFM images of a SWCNT assembly before and after treatment with an acidic solution $(\mathrm{pH} 2.0)$. All dimensions are in $\mathrm{nm}$.

an acidic solution, the surface roughness of the densely packed monolayer of standing SWCNTs decreases as the SWCNT layer is desorbed, thus resulting in a smooth surface. The adsorption-desorption processes of SWCNTs on different SAM-modified surfaces were followed by SPR spectroscopy (Figure S8a). The obtained data show that the original DTBPY-modified surface is recovered by the $\mathrm{pH}$-induced cleaning process; furthermore, the reusability of the SWCNTs adsorbed on a recycled substrate is comparable to that of SWCNTs supported on a fresh SAM-modified surface (Figure S8b). In contrast, only negligible amounts of SWCNTs are desorbed from PT and BPT-based assemblies treated with an acidic solution.

The recycling capability of DTBPY-modified substrates allows the regeneration of the device and the sequential assembly of SWCNTs onto the surface. These results demonstrate the reversibility of the $\pi-\pi$ stacking interactions between pyridine and SWCNTs and their strict dependence on the $\mathrm{pH}$ value of the solution. In general, the system represents a new, interesting recycling model.

To test the suitability of this system for practical applications, rhodamine-conjugated avidin was attached to a lithographically patterned gold surface through biotin-terminated SWCNTs. In the first step, a succinimide-terminated biotin linker was reacted with BPT to form bridging maleimide- active ester units. Biphenyl-terminated biotin was added onto the SWCNT-modified surface and incubated for $2 \mathrm{~h}$. The terminal biotin groups that were thus assembled on SWCNTmodified surfaces were subsequently reacted with fluorescent-labeled avidin (see the Supporting Information for more details). The biotin-avidin linkage is a strong biological interaction (binding constant of $10^{-15} \mathrm{M}^{-1}$ ), which shows high stability over a broad $\mathrm{pH}$ range. ${ }^{[17]}$ The initial fluorescence image of Figure $4 \mathrm{a}$ shows that the SWCNT-modified $\mathrm{Au}$ surfaces are uniformly functionalized with avidin.
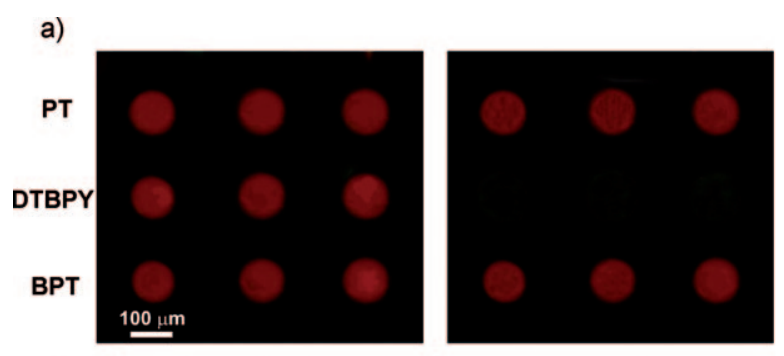

b)
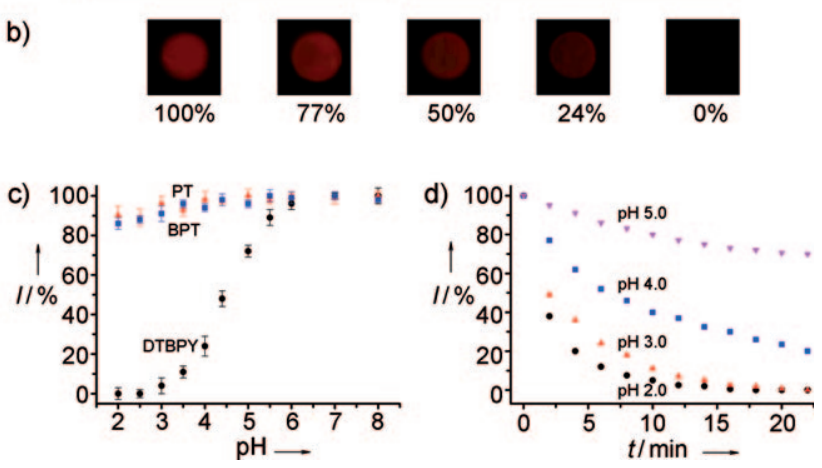

Figure 4. a) Fluorescence image of avidin-functionalized SWCNTs assembled on a gold array modified with a PT, DTBPY, and a BPT monolayer before and after treatment with an acidic solution $(\mathrm{pH} 2.0)$. b) Fluorescence images of an avidin-functionalized SWCNT surface as a function of coverage during desorption. c) Plot of fluorescence intensity versus $\mathrm{pH}$ value for avidin-modified SWCNTs assembled on PT, BPT, and DTBPY SAMs. The fluorescence was measured after 20 min of contact with the acidic solution. Error bars correspond to a set of $N=5$ measurements. d) Plot of fluorescence intensity versus desorption time for avidin-modified SWCNTs assembled on a DTBPY monolayer at different $\mathrm{pH}$ values.

After treatment with an acidic solution ( $\mathrm{pH} 2.0)$, the SWCNTs anchored on a DTBPY SAMs undergo desorption and diffuse into bulk solution, as evidenced by the complete loss of the fluorescence signal (Figure $4 \mathrm{a}$ ). Note that only the spots modified with DTBPY appear dark after the acidic treatment; those modified with PT and BPT still show red fluorescence.

Control experiments were carried out on unmodified SWCNTs assembled on SAMs as proteins tend to adsorb unspecifically on SWCNTs. These experiments indicate that the extent of avidin immobilized on SWCNTs through nonspecific binding is negligible when compared to the large amount of avidin present on biotin-functionalized SWCNTs. A further aspect relates to the effect of SWCNTs on the immobilization of biotin. The immobilization of biotin 
on an SWCNT-free SAM-modified gold substrate was studied by probing the fluorescence changes upon addition of avidin. No well-defined fluorescent spots were observed at different avidin concentrations.

The release kinetics of the protein-conjugated SWCNTs was evaluated by real time fluorescence imaging. Figure 4c shows a representative plot of the fluorescence associated with the avidin-modified SWCNTs assembly versus $\mathrm{pH}$ value. In the case of SWCNTs assembled on DTBPY, the onset of desorption occurs at a $\mathrm{pH}$ value of approximately 6.0 , and is complete at a $\mathrm{pH}$ value of approximately 2.5 , where the fluorescence signal intensity decreases to zero. The threshold $\mathrm{pH}$ value is lower than that expected from the $\mathrm{p} K_{\mathrm{a}}$ value of the DTBPY molecules as a result of the lower value of the apparent dissociation constant of the DTBPY molecule assembled in densely packed SAMs. ${ }^{[18]}$ Figure $4 \mathrm{~d}$ shows the dependence of the fluorescence signal intensity as a function of time and at different $\mathrm{pH}$ values for SWCNTs assembled on DTBPY monolayer. At pH 4.0 and $\mathrm{pH} 5.0$, the fluorescence intensity trend is consistent with a complex desorption phenomenon, which can be ascribed to a slow transport rate of the SWCNTs away from the surface. On the contrary, at low $\mathrm{pH}$ values (2.0 and 3.0), the fluorescence signal disappears after $10 \mathrm{~min}$, consistent with complete desorption of the protein-conjugated SWCNTs. By using this platform, biotinylated proteins and peptides can be tethered on SWCNTs and hence the patterning and release of the hybrid material can be readily controlled.

In conclusion, we have reported the controlled reversible assembly of SWCNTs on SAM-modified surfaces. The assembly is achieved by $\pi-\pi$ interactions that occur between the SWCNTs and aromatic rings that are anchored on the gold surface. The assembly of SWCNTs on aromatic SAMs results in highly oriented SWCNT arrays and increases the electronic coupling between a gold electrode surface and soluble redox species. This method is more simple and convenient with respect to covalent immobilization because of the possibility of recycling and reuse of the substrate. Moreover, we have demonstrated that the protonation of the DTBPY layer controls the DTBPY-SWCNTs $\pi-\pi$ interactions. This control enables an efficient $\mathrm{pH}$-stimulated release of SWCNTs from the gold surface to the aqueous solution. We believe that the $\mathrm{pH}$-controlled reversible assembly of SWCNTs holds great potential not only in fundamental research that requires spatially controlled release of SWCNTs, but also toward practical applications, such as the release of SWCNTs that deliver drugs or biomolecules to living cells at very low concentrations.

Received: April 7, 2011

Published online: June 17, 2011

Keywords: carbon nanotubes $\cdot$ monolayers $\cdot \pi-\pi$ interactions . self-assembly · surface chemistry

[1] S. J. Tans, A. R. M. Verschueren, C. Dekker, Nature 1998, 393, $49-52$.
[2] a) B. Pradhan, S. K. Batabyal, A. J. Pal, Appl. Phys. Lett. 2006, 88,093106 ; b) E. Maligaspe, A. S. D. Sandanayaka, T. Hasobe, O. Ito, F. D'Souza, J. Am. Chem. Soc. 2010, 132, 8158-8164; c) O. Ovits, R. Tel-Vered, I. Baravik, O. I. Wilner, I. Willner, J. Mater. Chem. 2009, 19, 7650-7655; d) G. Bottari, G. de La Torre, D. M. Guldi, T. Torres, Chem. Rev. 2010, 110, 6768-6816.

[3] a) J. J. Gooding, Electrochim. Acta 2005, 50, 3049-3060; b) J. Wang, Electroanalysis 2005, 17, 7-14.

[4] a) E. Katz, I. Willner, ChemPhysChem 2004, 5, 1084-1104; b) Y.-M. Yan, O. Yehezkeli, I. Willner, Chem. Eur. J. 2007, 13, 10168-10175; c) A. Callegari, S. Cosnier, M. Marcaccio, D. Paolucci, F. Paolucci, V. Georgakilas, N. Tagmatarchis, E. Vázquez, M. Prato, J. Mater. Chem. 2004, 14, 807-810; d) X. Yu, B. Munge, V. Patel, G. Jensen, A. Bhirde, J. D. Gong, S. N. Kim, J. Gillespie, J. S. Gutkind, F. Papadimitrakopoulos, J. F. Rusling, J. Am. Chem. Soc. 2006, 128, 11199-11205; e) S. N. Kim, J. F. Rusling, F. Papadimitrakopoulos, Adv. Mater. 2007, 19, 3214-3228; f) J. Wang, G. Liu, M. R. Jan, J. Am. Chem. Soc. 2004, 126, 3010-3011; g) A. Le Goff, M. Holzinger, S. Cosnier, Analyst 2011, 136, 1279-1287.

[5] a) D. Tasis, N. Tagmatarchis, A. Bianco, M. Prato, Chem. Rev. 2006, 106, 1105-1136; b) Y.-L. Zhao, J. F. Stoddart, Acc. Chem. Res. 2009, 42, 1161-1171; c) N. Karousis, N. Tagmatarchis, D. Tasis, Chem. Rev. 2010, 110, 5366-5397.

[6] a) F. Hauquier, G. Pastorin, P. Hapiot, M. Prato, A. Bianco, B. Fabre, Chem. Commun. 2006, 4536-4538; b) D. J. Garrett, B. S. Flavel, J. G. Shapter, K. H. R. Baronian, A. J. Downard, Langmuir 2010, 26, 1848-1854; c) P. Viel, X. Tuan Le, V. Huc, J. Bar, A. Benedetto, A. Le Goff, A. Filoramo, D. Alamarguy, S. Noël, L. Baratone, S. Palacin, J. Mater. Chem. 2008, 18, 5913-5920.

[7] a) V. C. Moore, M. S. Strano, E. H. Haroz, R. H. Hauge, R. E. Smalley, J. Schmidt, T. Talmor, Nano Lett. 2003, 3, 1379-1383; b) B. K. Price, J. L. Hudson, J. M. Tour, J. Am. Chem. Soc. 2005, 127, 14867-14871; c) S.-Y. Ju, M. Utz, F. Papadimitrakopoulos, J. Am. Chem. Soc. 2009, 131, 6775-6784; d) M. Holzinger, J. Baur, R. Haddad, X. Wang, S. Cosnier, Chem. Commun. 2011, $47,2450-2452$.

[8] a) A. Star, D. Steuerman, J. R. Heath, J. F. Stoddart, Angew. Chem. 2002, 114, 2618-2622; Angew. Chem. Int. Ed. 2002, 41, 2508-2512; b) Y.-M. Yan, I. Baravik, O. Yehezkeli, I. Willner, J. Phys. Chem. C 2008, 112, 17883-17888; c) S. Cosnier, R. E. Ionescu, M. Holzinger, J. Mater. Chem. 2008, 18, 5129-5133; d) J. Wang, M. Musameh, Y. Lin, J. Am. Chem. Soc. 2003, 125, $2408-2409$; e) L. Hu, D. S. Hecht, G. Grüner, Chem. Rev. 2010, $110,5790-5844$.

[9] a) A. Chou, T. Bocking, N. K. Singh, J. J. Gooding, Chem. Commun. 2005, $842-844$; b) C. E. Banks, T. J. Davies, G. G. Wildgoose, R. G. Compton, Chem. Commun. 2005, 829-841; c) W. Yang, K. R. Ratinac, S. P. Riner, P. Thordarson, J. J. Gooding, F. Braet, Angew. Chem. 2010, 122, 2160-2185; Angew. Chem. Int. Ed. 2010, 49, 2114-2138; d) P. Diao, Z. Liu, $A d v$. Mater. 2010, 22, 1430-1449; e) T. Druzhinina , S. Hoeppener, U. S. Schubert, Adv. Mater. 2011, 23, 953-970.

[10] a) Z. Liu, Z. Shen, T. Zhu, S. Hou, L. Ying, Langmuir 2000, 16, 3569-3573; b) P. Diao, Z. Liu, B. Wu, X. Nan, J. Zhang, Z. Wei, ChemPhysChem 2002, 3, 898-901; c) J. J. Gooding, R. Wibowo, J. Liu, W. Yang, D. Losic, S. Orbons, F. J. Maarns, J. G. Shapter, D. B. Hibbert, J. Am. Chem. Soc. 2003, 125, 9006-9007; d) F. Patolsky, Y. Weizmann, I. Willner, Angew. Chem. 2004, 116, 2165-2169; Angew. Chem. Int. Ed. 2004, 43, 2113-2117; e) J. J. Gooding, A. Chou, J. Liu, D. Losic, J. G. Shapter, D. B. Hibbert, Electrochem. Commun. 2007, 9, 1677-1683; f) L. Cai, J. L. Bahr, Y. Yao, J. M. Tour, Chem. Mater. 2002, 14, 4235-4241; g) A. Chou, P. K. Eggers, M. N. Paddon-Row, J. J. Gooding, J. Phys. Chem. C 2009, 113, 3203-3211. 


\section{Communications}

[11] O. Arias de Fuentes, T. Ferri, M. Frasconi, V. Paolini, R. Santucci, Angew. Chem. 2011, 123, 3519-3523; Angew. Chem. Int. Ed. 2011, 50, 3457-3461.

[12] a) D. Chattopadhyay, I. Galeska, F. Papadimitrakopoulos, J. Am. Chem. Soc. 2001, 123, 9451-9452; b) B. Wu, J. Zhang, Z. Wei, S Cai, Z. Liu, J. Phys. Chem. B 2001, 105, 5075-5078.

[13] J. Liu, M. J. Casavant, M. Cox, D. A. Walters, P. Boul, W. Lu, A. J. Rimberg, K. A. Smith, D. T. Colbert, R. E. Smalley, Chem. Phys. Lett. 1999, 303, 125-129.

[14] G. S. Duesberg, I. Loa, M. Burghard, K. Syassen, S. Roth, Phys. Rev. Lett. 2000, 85, 5436-5439.
[15] W. Hu, Z. Lu, Y. Liu, C. M. Li, Langmuir 2010, 26, 8386-8391. [16] P. Chen, M. A. Fryling, R. L. McCreery, Anal. Chem. 1995, 67, 3115-3122.

[17] E. P. Diamandis, T. K. Christopoulos, Clin. Chem. 1991, 37, 625636.

[18] a) J. Liu, B. Schüpbach, A. Bashir, O. Shekhah, A. Nefedov, M. Kind, A. Terfort, C. Wöll, Phys. Chem. Chem. Phys. 2010, 12, 4459-4472; b) C. Silien, M. Buck, G. Goretzki, D. Lahaye, N. R. Champness, T. Weidner, M. Zharnikov, Langmuir 2009, 25, 959 967. 